\title{
Somatic Anxiety May Represent Archers' Actual Autonomic Nervous System Activity But How: Moderating Role of Personality Traits
}

\author{
Nihal Dal ${ }^{1}$, Serdar Tok ${ }^{1}$, Erman Doğan ${ }^{2, *}$, İlker Balıkçı1 ${ }^{1}$, Aylin Zekioğlu ${ }^{1}$, Fatih Çatıkkaş ${ }^{1}$ \\ ${ }^{1}$ Faculty of Sport Sciences, Celal Bayar University, Turkey \\ ${ }^{2}$ School of Physical Education and Sports, Girne American University, Cyprus
}

Copyright $\bigcirc 2018$ by authors, all rights reserved. Authors agree that this article remains permanently open access under the terms of the Creative Commons Attribution License 4.0 International License

\begin{abstract}
Objective: In the present study we aimed to examine the relationship between competitive anxiety, autonomic nervous system activity represented by Heart Rate Variability (HRV) and respiration, and performance in novice archers. We also interested in whether neuroticism may be a factor with a potential to moderate the link among anxiety, autonomic activity and archers' performance. Methods: The sample included 39 novice archers ranging in age from 18 to 27. Participants first completed to anxiety and neuroticism measures. Then, participants' HRV and respiration activity was measured for 4 minutes. Afterwards, participants shot 10 arrows from $18 \mathrm{~m}$ to an $80 \mathrm{~cm}$ diameter target while their HRV and respiration was measured. Shooting accuracy was determined in terms of radial distance (error) from the center of the target. To determine an overall performance score, the median value of the 10 arrows was calculated for each participant. Spearman correlation analyses together with linear regression were used to analyze obtained data set. Results: Overall, results indicated an association between respiration and anxiety. Moreover, results provided preliminary evidence that neuroticism may be a factor having potential to moderate the relationship between anxiety and autonomic activity before and during an archery competition. Discussion: Results of the present study suggested that determination of archers' performance should be based upon both physiological and psychological factors.
\end{abstract}

Keywords Anxiety, Heart Rate Variability, Respiration, Neuroticism, Archery

\section{Introduction}

Archery is a unique athletic discipline in which psychological state such as anxiety and related autonomic responses may have a vital influence on performance. Therefore, there is a clear need to understand archers' psycho-physiological responses and factors with a potential to affect these responses.

The Multidimensional Anxiety Theory (MAT) (Martens, Burton, Vealey, Bump \& Smith, 1990) is one of the most influential theoretical frameworks providing a comprehensive scientific ground for the researchers aiming to clarify the relationship anxiety and athletic performance. Briefly stated, MAT considers anxiety as a multidimensional construct consisting of both somatic and cognitive subcomponents. The MAT defines cognitive anxiety as "the mental component of anxiety and is caused by negative expectations about success or by negative self-evaluation" (Martens et al., 1990, p.6) and a negative linear relationship is expected between cognitive anxiety and performance. On the other hand, Martens et al. (1990, p. 6) defined somatic anxiety as "the physiological and affective elements of the anxiety experience that develop directly from autonomic arousal" and they suggested an inverted U shaped relationship between somatic anxiety and performance. In addition to anxiety, Martens at al., (1990) considers self-confidence as a third factor having a potential to have positive influence on performance, although, it is not an original anxiety component.

To date, the MAT has attracted incredible amount of scientific attention from the researcher in the field of sport psychology (Hagan, Pollmann, \& Schack, 2017) (Bum \& Shin, 2015) . In this studies researchers' efforts focused mainly on the examination of MAT's hypothesis regarding the relationship between anxiety subcomponents and athletic performance (Robazza \& Bortoli, 2007; Pijpers, Oudejans, Holsheimer, \& Bakker, 2003; Parry, Chinnasamy, Papadopoulou, Noakes, \& Micklewright, 2011; Sanchez, Boschker, \& Llewellyn, 2010). Researchers also examined suggested anxiety components 
in relation to other psychological constructs such as perfectionism (Martinent \& Ferrand, 2007), self-esteem (Koivula \& Hassme, 2002) and motivational climate (Smith, Smoll, \& Cumming, 2007).

Given the wealth of study examining MAT's prediction, there are still some theoretical issues that need to be addressed. In this respect, only few study examined whether somatic anxiety may be associated actual physiological arousal measured by objective methods. Further, Woodman and Hardy (2003) stated that "somatic anxiety is of little theoretical value in explaining the relationship between physiological arousal and performance" (p. 444). Therefore, examination of somatic anxiety in relation to autonomic nervous system activity is of great importance and may have potential to contribute to our understanding of the relationship between somatic anxiety and actual physiological or autonomic arousal.

Heart Rate Variability (HRV) is one of the most prominent noninvasive indicator of autonomic nervous system and according to Carrillo, Christodoulou, Koutedakis, and Flouris (2011) HRV might be a useful tool to explore autonomic nervous system activity during a precision task. The autonomic nervous system is modulated by two well balanced systems. The Sympathetic Nervous System (SNS), which is related to the fight or flight response; and the Parasympathetic Nervous System (PNS), which is associated with rest and the digestive system (Thayer \& Lane, 2009). In accordance with Massimo Pagani's (Malliani, Pagani, Lombardi, \& Cerutti, 1991; Montano et al., 2009; Pagani et al., 1986) model, it is accepted that three components may represent SNS, PNS and balanced between them. Therefore, High Frequency (HF) power $(0.15$ to $0.40 \mathrm{~Hz})$ is recognized as an indicator of cardiac parasympathetic tone. On the other hand, Low Frequency (LF) power (0.04 to 0.15$)$ is accepted as a marker of cardiac sympathetic outflow. Sympathovagal tone is determined by the LF/HF ratio (Reyes del Paso, Langewitz, Mulder, van Roon, \& Duschek, 2013). Previous studies in sport psychology as well as other fields of psychology provided robust evidence that HRV could be an indicator mental state (Miu, Heilman, \& Miclea, 2009; Cervantes Blásquez, Rodas Font, \& Capdevila Ortís, 2009). Therefore, HRV can be considered as a psycho-physiological parameter having potential to reflect autonomic nervous system arousal triggered by mental state. Another indicator of autonomic activity is the respiration rate which is also vital for the shooting accuracy in archery. Previously, it has been suggested that respiratory activity is sensitive to variety of psychological variables (Boiten, F. A., Frijda, N. H., \& Wientjes, 1994; Lorig, 2007). Although, to our best of knowledge, no previous study examined the link between anxieties and related respiratory responses in archers, the aforementioned arguments and research findings may provide a theoretically sound ground to examine this association in archers.
Another issue that must be dealt with is the lack of understanding regarding whether certain individual differences may have potential to moderate the relationship between anxiety, autonomic responses and performance. In this regard, considering the argument by Ebstrup, Eplov, Pisinger, and Jørgensen (2011) suggesting that personality traits, especially those within the Big Five personality model, may give rise to different methods of appraising and coping stress, it can be concluded that personality traits may lead better understanding of the association among anxiety, autonomic responses and performance. The most important personality traits that can alter athletes' autonomic responses to anxiety before and during the execution of a motor is the neuroticism. Neuroticism may create a disadvantage in tolerance of the debilitating effects of stress on performance because of low arousability of the limbic.

System (Matthews \& Gilliland, 1999). Therefore, athletes having higher level of neuroticism may be more predispose to experience anxiety and physiological arousal.

In the present study we aimed to explore the relationship between somatic and cognitive anxiety, related autonomic activity represented by HRV, respiration and performance in archers. Based on the theoretical frameworks and research findings mentioned above we postulated that physiological arousal should be associated with anxiety components both before and during an important archery event. Moreover, this relationship should be more evident among the athletes having higher level of neuroticism.

\section{Material and Methods}

\subsection{Participants}

Participants were 39 (12 female) college students ranging in age from 18 to 27 [23.5 (2.11), mean (s)]. None of the participants had previous experience in archery. However, they enrolled and completed in a 14-week archery class to receive course credit. Participants had no acute or chronic neuromuscular disease or psychiatric disorder and were required to abstain from the use of any medications that may affect nervous system functioning.

\subsection{Personality Measures}

The short form of the Five Factor Personality Inventory (FFPI) developed by Tatar (2005) was used to assess conscientiousness; this is an 85 -item personality inventory designed to evaluate the five main personality traits, namely, neuroticism, extraversion, openness to experience, agreeableness and conscientiousness. Item responses are made using a 5-point format. In this study, only the neuroticism subscale of the FFPI was used. Internal consistency score for the neuroticism factor was 0.87 . 


\subsection{Anxiety Measures}

The Competitive State Anxiety Inventory-2 (CSAI-2) (Martens, et al., 1990) was used to measure competitive anxiety. The inventory contains 27 items, nine for each of the three subscales of Self-Confidence, Cognitive Anxiety, and Somatic Anxiety. The 27 items were measured using a Likert-type scale ranging from 1 (not at all) to 4 (very much so). Cronbach's alpha coefficients were .80 for Self-Confidence, .77 for Cognitive Anxiety, and .82 for Somatic Anxiety.

\subsection{Procedure}

In the first stage of the experiment, participants first completed the CSAI-2. Then, their resting HRV was measured for 4 minutes while sitting. In the next stage, participants shot 10 arrows in 4 minutes from $18 \mathrm{~m}$ to an $80-\mathrm{cm}$ diameter target. The shooting task was self-paced, so participants decided when to shoot an arrow and how long to prepare to shoot. Based on a pilot study of five novice archers, we determined that participants were required to shoot an arrow in approximately 20-25 seconds. Participants were given additional arrows if they finished their shooting in less than 4 minutes. However, only the first 10 shots were taken into account. Performance was determined as the sum of the first 10 shots.

\subsection{Statistical Analyses}

To explore the relations of CSAI-2 components, namely cognitive anxiety, somatic anxiety and self-confidence, to shooting performance, respiration rate and HRV measured during before and during shooting session Spearman rho correlation coefficient were calculated. Afterwards, the sample was partitioned based on a median split of the neuroticism scores as high and low neuroticism groups. Then the same relation correlation analyses were repeated for both groups. Finally, we tested whether the regression model containing CSAI-2 components may have ability to predict performance and physiological arousal represented as respiration in both low and high neuroticism groups.

\section{Results}

Considering the entire sample results of Spearman rho correlation showed that among the CSAI-2 components cognitive $(\mathrm{r}=.49, \mathrm{p}=.002)$ and somatic anxiety $(\mathrm{r}=.35, \mathrm{p}$ $=.003)$ were positively and significantly related to respiration rate measured before shooting session. However, self-confidence $(\mathrm{r}=-.33, \mathrm{p}=.041)$ was negatively and significantly correlated to respiration rate. None of the CSAI-2 components were associated HRV measured before the shooting session. The relation between CSAI-2 components was also unrelated to shooting performance.
We also interested in whether association between CSAI-2 components, psycho-physiological arousal and performance may be differed as result of neuroticism. Hence, the sample was partitioned based on a median split of the neuroticism scores as high and low neuroticism groups and the same relationships were examined in these groups. Results demonstrated that in high neuroticism group cognitive anxiety was positively associated with respiration $(\mathrm{r}=.58, \mathrm{p}=.009)$ measured before shooting session and negatively associated with shooting performance $(r=-.62, p=.005)$. There was no other significant correlation in high neuroticism group. In low neuroticism group, cognitive anxiety was significantly associated HF power $(r=-.49, p=.047)$ measured before shooting session. No other significant association was found among CSAI- components, psycho-physiological arousal and performance in low neuroticism group.

The link among CSAI-2 components, psycho-physiological arousal measured during shooting and shooting performance was also examined. Results indicated that cognitive anxiety was significantly correlated to respiration $(r=-.43, p=.006)$ in entire sample. Neither, somatic anxiety nor self-confidence was associated with psycho-physiological arousal and performance. When the sample partitioned as high and low neuroticism groups results demonstrated that self-confidence component of was inversely and significantly related to LF power $(\mathrm{r}=-.53, \mathrm{p}=.017)$ in low neuroticism group. There was no other significant relationship in low neuroticism group. In high neuroticism group, both cognitive $(\mathrm{r}=-.55, \mathrm{p}=.015)$ and somatic anxiety $(r=-.47, p=.043)$ has been found to be negatively and significantly associated with $\mathrm{LF} / \mathrm{HF}$ ratio measured during the shooting session. Cognitive anxiety $(r=.73, p$ $=.001$ ) was also correlated to respiration measured during the shooting session in high neuroticism group. In high neuroticism group, respiration measured during the shooting session was inversely related to shooting performance $(\mathrm{r}=-.59, \mathrm{p}=.008)$.

Based on the significant associations between CSIA-2 components, psycho-physiological arousal, especially respiration, we decided to test whether the regression model consisting of the CSAI-2 components of cognitive anxiety, somatic anxiety and self-confidence may have ability to predict respiration measured during shooting session and the shooting performance in high and low neuroticism groups. In low neuroticism group, results revealed that the model failed to explain significant amount of variance in respiration measured during shooting session $\left[\mathrm{F}(3,16)=.812, \mathrm{P}=.506, \mathrm{R}=.13 \mathrm{R}_{\text {adj }}^{2}=-.031\right]$ and shooting performance $[\mathrm{F}(3,16)=.995, \mathrm{P}=.421 \mathrm{R}=.15$, $\left.\mathrm{R}^{2}{ }_{\mathrm{adj}}=-.001\right]$. On the other hand, the same model was able to account significant amount of variance in respiration measured during the shooting session and $[\mathrm{F}(3,15)=.5 .33$, $\left.\mathrm{P}=.011, \mathrm{R}=.52 \mathrm{R}_{\text {adj }}^{2}=.42\right]$ performance $[\mathrm{F}(3,15)=3.58$, $\left.\mathrm{P}=.039, \mathrm{R}=.41 \mathrm{R}^{2}{ }_{\text {adj }}=.30\right]$. 


\section{Discussion}

In the present study we aimed to examine the relationship among somatic and cognitive anxiety, related autonomic activity represented by HRV and respiration, and performance in archers. We also interested in whether aforementioned association may vary as a result of neuroticism.

Results of the study provided some evidence for our predictions regarding the relationship among anxiety, related autonomic activity and performance. Hence, we found that both somatic and cognitive anxiety were positively associated with the respiration in entire sample. However, respiration was more strongly associated with cognitive anxiety than somatic anxiety. Considering CSAI-2 items measuring cognitive anxiety such as "I am concerned about losing" one would not to find a connection between cognitive anxiety and autonomic nervous system activity. Previously, Alix-Sy, Le Scanff, \& Filaire, (2008) found somatic anxiety to be associated with salivary cortisol level which provides partial support for results. On the other hand, no previous study provided results whether cognitive anxiety may be associated with actual physiological arousal. Therefore, in future studies researchers should consider whether cognitive anxiety in addition to somatic anxiety may be a useful construct to understand athletes' actual physiological arousal. Contrary to anxiety, self-confidence has been found to be inversely correlated to the respiration. This result indicated that in addition to anxiety components of CSIA-2, self-confidence may also be correlated to physiological arousal.

Another result that must be dealt with is the lack of association between HRV and anxiety components. Considering entire sample, neither cognitive anxiety nor somatic anxiety was associated with HRV. This result led us to argue that cognitive and somatic anxiety may be associated only certain psycho-physiological parameters.

We found cognitive and somatic anxiety to be unrelated to performance (shooting accuracy in this case) in entire sample. Similarly, considering entire sample self-confidence was also unrelated to performance. Examination of the two previous extensive meta-analyses on the relation of anxiety and confidence as measured by CSAI-2 to performance yielded contradictory results. In this respect, Woodman and Hardy (2003) provided support for the suggested association of cognitive anxiety and confidence with performance. Contrary, the other meta-analysis by Craft, Magyar, Becker, and Feltz (2003) found only weak association between the same variables. However, it should be noted that neither meta-analysis included studies examining the link between anxiety, confidence and performance in archery. Hence, it seems that there is a clear need to explore this issue on this specific population.

The last issue that we want to address is the results showing that the association between anxiety, autonomic activity and performance may vary as result of neuroticism. Accordingly, LF power which is an indicator of sympathetic nervous system and sensitive to both physical (Martinmäki \& Rusko, 2008) and mental load (Luft, Takase, \& Darby, 2009; Park, Vasey, Van Bavel, \& Thayer, 2013) was inversely correlated to LF power only in low neuroticism group. This result may provide a preliminary evidence for the argument that self-confidence may be a factor having potential to buffer sympathetic cardiac activity only in archers with a lower level neuroticism. In other word, neuroticism may be mediating factor for the link between self-confidence and sympathetic cardiac activity which may have a vital effect on archers' shooting precision. In high neuroticism group, both cognitive and somatic anxiety was inversely and significantly correlated to $\mathrm{LF} / \mathrm{HF}$ ratio which is a parameter that reflects the comparative balance of the two branches of the autonomic nervous system on cardiac activity (Kao et al., 2016). In fact, contrary to the results obtained in the present study a positive relationship between competitive anxiety and $\mathrm{LF} / \mathrm{HF}$ ratio is expected (Cervantes Blásquez et al., 2009). However, it should be noted archers should be able to strictly control their respiration activity for a more precise arrow shooting. In addition, archery is also a physically demanding athletic discipline and requires appreciable amount of muscular strength. Hence, during an arrow shooting session an increase in sympathetic cardiac activity together with a parasympathetic withdrawal (greater $\mathrm{LF} / \mathrm{HF}$ ratio) is expected. Therefore, we concluded that the negative correlation between anxiety and LF/HF ratio, indicating relatively higher sympathovagal balance, in high neuroticism group can be an evidence that these archers failed to restrict their respiration activity during the arrow shooting. Strong positive $(r=.73, p=.001)$ association between cognitive anxiety and respiration only in high neuroticism group provide support for our conclusion.

The present study may have several implications for both researchers and practitioners such as Olympic archery trainers and athletes. First, our results provide a preliminary evidence that neuroticism within the Big Five personality model can give rise to different autonomic cardiac responses in archers. Such an information may be helpful for the selection of prospective archery athletes. Second, ability to control the respiration may be influenced by the neuroticism.

\section{REFERENCES}

[1] Alix-Sy, D., Le Scanff, C., \& Filaire, E. (2008). Psychophysiological responses in the pre-competition period in elite soccer players. Journal of Sports Science and Medicine, 7(December), 446-454.

[2] Boiten, F. A., Frijda, N. H., \& Wientjes, C. J. (1994). Emotions and respiratory patterns: review and critical 
analysis. International Journal of Psychophysiology, 17(2), 103-128.

[3] Bum, C.-H., \& Shin, S. H. (2015). The Relationships between Coaches' Leadership Styles, Competitive State Anxiety, and Golf Performance in Korean Junior Golfers Sport Science Review. 24(5-6), 371-386. https://doi.org/https://doi.org/10.1515/ssr-2015-0024

[4] Carrillo, A. E., Christodoulou, V. X., Koutedakis, Y., \& Flouris, A. D. (2011). Autonomic nervous system modulation during an archery competition in novice and experienced adolescent archers. Journal of Sports Sciences, 29(June), 913-917. https://doi.org/10.1080/02640414.2011 .568514

[5] Cervantes Blásquez, J. C., Rodas Font, G., \& Capdevila Ortís, L. (2009). Heart-rate variability and precompetitive anxiety in swimmers. Psicothema, 21, 531-536.

[6] Craft, L. L., Magyar, T. M., Becker, B. J., \& Feltz, D. L. (2003). The relationship between the Competitive State Anxiety Inventory-2 and sport performance: a meta-analysis. Journal of Sport \& Exercise Psychology, 25 , 44-65. Retrieved from http://articles.sirc.ca/search.cfm?id $=\mathrm{S}-880535 \&$ scope $=$ cite $\% 5 \mathrm{Cnhttp}: / /$ content.ebscohost.com. faraway.u-paris 10. fr/ContentServer.asp? $\mathrm{T}=\mathrm{P} \& \mathrm{P}=\mathrm{AN} \& \mathrm{~K}=$ SPHS-880535\&S $=\mathrm{R} \& \mathrm{D}=\mathrm{s} 3 \mathrm{~h} \&$ EbscoContent $=\mathrm{dGJyMNLr}$ 40Sep7A4yOvsOLCmr0qep7BSsKu4TLSWxWXS\&Cont entCustomer=dGJyMPGntkiyr7NJuePfg

[7] Ebstrup, J. F., Eplov, L. F., Pisinger, C., \& Jørgensen, T. (2011). Association between the Five Factor personality traits and perceived stress: is the effect mediated by general self-efficacy? Anxiety, Stress, \& Coping, 24(4), 407-419. https://doi.org/10.1080/10615806.2010.540012

[8] Hagan, J. E., Pollmann, D., \& Schack, T. (2017). Interaction between Gender and Skill on Competitive State Anxiety Using the Time-to-Event Paradigm: What Roles Do Intensity, Direction, and Frequency Dimensions Play? Frontiers in Psychology. Retrieved from https://www.front iersin.org/article/10.3389/fpsyg.2017.00692

[9] Kao, L. C., Liu, Y. W., Tzeng, N. S., Kuo, T. B. J., Huang, S. Y., Chang, C. C., \& Chang, H. A. (2016). Linking an anxiety-related personality trait to cardiac autonomic regulation in well-defined healthy adults: Harm avoidance and resting heart rate variability. Psychiatry Investigation, 13(4), 397-405. https://doi.org/10.4306/pi.2016.13.4.397

[10] Koivula, N., \& Hassme, P. (2002). Self-esteem and perfectionism in elite athletes: effects on competitive anxiety and self-confidence, $32,865-875$.

[11] Lorig, T. S. (2007). No Title. In G. G. Cacioppo, J. T., Tassinary, L. G., Berntson (Ed.), Handbook of Psychophysiology (Third Edit, pp. 231-245). Cambridge: Cambridge University Press.

[12] Luft, C. D. B., Takase, E., \& Darby, D. (2009). Heart rate variability and cognitive function: Effects of physical effort. Biological Psychology, 82(2), 186-191. https://doi.org/10. 1016/j.biopsycho.2009.07.007

[13] Malliani, a, Pagani, M., Lombardi, F., \& Cerutti, S. (1991). Cardiovascular neural regulation explored in the frequency domain. Circulation, 84(2), 482-492. https://doi.org/10.11 61/01.CIR.84.2.482
[14] Martens, R., Burton, D., Vealey, R.S., Bump, L. A. and, \& Smith, D. E. (1990). Development and validation of the Competitive State Anxiety Inventory-2. In R. S. V. and D. B. R. Martens (Ed.), Competitive Anxiety in Sport (pp. 117190). Champaign, IL: Human Kinetics.

[15] Martinent, G., \& Ferrand, C. (2007). A cluster analysis of precompetitive anxiety: Relationship with perfectionism and trait anxiety. Personality and Individual Differences, 43(7), 1676-1686. https://doi.org/10.1016/j.paid.2007.05.0 05

[16] Martinmäki, K., \& Rusko, H. (2008). Time-frequency analysis of heart rate variability during immediate recovery from low and high intensity exercise. European Journal of Applied Physiology, 102(3), 353-360. https://doi.org/10.10 07/s00421-007-0594-5

[17] Matthews, G., \& Gilliland, K. (1999). The personality theories of H. J. Eysenck and J. A. Gray: A comparative review. Personality and Individual Differences, 26(4), 583626. https://doi.org/10.1016/S0191-8869(98)00158-5

[18] Miu, A. C., Heilman, R. M., \& Miclea, M. (2009). Reduced heart rate variability and vagal tone in anxiety: Trait versus state, and the effects of autogenic training. Autonomic Neuroscience: Basic and Clinical, 145(1-2), 99-103. https://doi.org/10.1016/j.autneu.2008.11.010

[19] Montano, N., Porta, A., Cogliati, C., Costantino, G., Tobaldini, E., Casali, K. R., \& Iellamo, F. (2009). Heart rate variability explored in the frequency domain: A tool to investigate the link between heart and behavior. Neuroscience \& Biobehavioral Reviews, 33(2), 71-80. https://doi.org/10.1016/j.neubiorev.2008.07.006

[20] Pagani, M., Lombardi, F., Guzzetti, S., Rimoldi, O., Furlan, R., Pizzinelli, P., ... Piccaluga, E. (1986). Power spectral analysis of heart rate and arterial pressure variabilities as a marker of sympatho-vagal interaction in man and conscious dog. Circulation Research, 59(2), 178-193. https://doi.org/ 10.1161/01.RES.59.2.178

[21] Park, G., Vasey, M. W., Van Bavel, J. J., \& Thayer, J. F. (2013). Cardiac vagal tone is correlated with selective attention to neutral distractors under load. Psychophysiology, 50(4), 398-406. https://doi.org/10.1111 /psyp.12029

[22] Parry, D., Chinnasamy, C., Papadopoulou, E., Noakes, T., \& Micklewright, D. (2011). Cognition and performance: anxiety, mood and perceived exertion among Ironman triathletes. $\mathrm{Br} J$ Sports Med, 45(14), 1088-1094. https://doi.org/10.1136/bjsm.2010.072637

[23] Pijpers, J. R., Oudejans, R. R. D., Holsheimer, F., \& Bakker, F. C. (2003). Anxiety-performance relationships in climbing: A process-oriented approach. Psychology of Sport and Exercise, 4(3), 283-304. https://doi.org/10.1016/ S1469-0292(02)00010-9

[24] Reyes del Paso, G. a., Langewitz, W., Mulder, L. J. M., van Roon, A., \& Duschek, S. (2013). The utility of low frequency heart rate variability as an index of sympathetic cardiac tone: A review with emphasis on a reanalysis of previous studies. Psychophysiology, 50(5), 477-487. https://doi.org/10.1111/psyp.12027

[25] Robazza, C., \& Bortoli, L. (2007). Perceived impact of anger and anxiety on sporting performance in rugby players. 
Psychology of Sport and Exercise, 8(6), 875-896. https://doi.org/10.1016/j.psychsport.2006.07.005

[26] Sanchez, X., Boschker, M. S. J., \& Llewellyn, D. J. (2010). Pre-performance psychological states and performance in an elite climbing competition. Scandinavian Journal of Medicine and Science in Sports, 20(2), 356-363. https://doi.org/10.1111/j.1600-0838.2009.00904.x

[27] Smith, R. E., Smoll, F. L., \& Cumming, S. P. (2007). Effects of a motivational climate inntervention for coaches on young athletes' sport performance anxiety. Journal of Sport \& Exercise Psychology, 29(1), 39-59.

[28] Thayer, J. F., \& Lane, R. D. (2009). Claude Bernard and the heart-brain connection: Further elaboration of a model of neurovisceral integration. Neuroscience \& Biobehavioral Reviews, 33(2), 81-88.https://doi.org/10.1016/j.neubiorev. 2008.08.004

[29] Woodman, T., \& Hardy, L. (2003). The relative impact of cognitive anxiety and self-confidence upon sport performance: a meta-analysis. Journal of Sports Sciences, 21(6), 443-457. https://doi.org/10.1080/0264041031000101809

[30] Woodman, T. I. M., \& Hardy, L. E. W. (2003). The relative impact of cognitive anxiety and self-confidence upon sport performance: a meta-analysis, (1990), 443-457. https://doi.org/10.1080/0264041031000101809 